\title{
Experimental Method for Determination of Virtual Inertia Matrix Using Multivariate Regression Analysis
}

\author{
Koji Fukami and Shin-Ichiro Higashino* \\ Department of Aeronautics and Astronautics, Kyushu University, \\ 744 Moto-oka, Nishi-ku, Fukuoka 819-0395, Japan
}

(Received October 26, 2019; accepted December 7, 2019)

Keywords: inertia matrix, added mass, regression analysis, unmanned aerial vehicle

We present the concept of an experimental method for the determination of the inertia matrix with an added mass effect. This experimental method can be applied to any size and type of object. In particular, it can be most effectively used for small unmanned aerial vehicles (UAVs). The model vehicle is suspended by two thin light weight low-stretch lines vertically attached to two hook points on the model and provided with a free rotational oscillation to estimate the moment of inertia around the vertical axis through the center of gravity. This procedure is repeated at different attitude angles so that the moments of inertia around different axes can be calculated and used in the estimation of all components of the moments and products of inertia in the form of an inertia matrix, using a multivariate regression equation.

\section{Introduction}

There are an increasing number of industrial applications of remote sensing technologies using robots. An unmanned aerial vehicle (UAV) is an aircraft without a human pilot on board and has been increasingly used for various purposes and missions such as observation, measurement, inspection, transport, and maintenance in various industries. In general, equations of motion of the aircraft consist of aerodynamic terms on the right-hand side and inertia terms on the left-hand side, both of which are equally important in the design and analysis of flight control systems. There are a great number of studies on aerodynamic models; however, there are only a few studies on how to estimate the moments and products of inertia. As for small UAVs, it is highly likely that the moments and products of inertia predicted from the drawings or 3D CAD data are not sufficiently accurate because of the complicated shapes of adhesive components and electronic devices. Furthermore, what is actually needed for the lefthand side of the equations of motion is not just the inertia of a mass but the sum of the general mass and the added mass, which is called a virtual mass. The added mass is an inertia added to an object originating from some volume of surrounding fluid moving together with the object and is normally represented by a second-order tensor to relate the acceleration vector to the external force vector. Namely, it can be considered that what is needed for the design of a flight control system is a virtual inertia matrix.

\footnotetext{
*Corresponding author: e-mail: tonton@aero.kyushu-u.ac.jp
} https://doi.org/10.18494/SAM.2019.2665 
The measurement methods of the moment of inertia can be classified into two groups: ${ }^{(1)}$ a) the free oscillation technique is an experimental method of measuring the moment of inertia, ${ }^{(2-8)}$ where the model is mounted on a suspension device and then provided with a free oscillation; b) the forced oscillation technique is another method to measure the moment of inertia, ${ }^{(9-11)}$ where the model is provided with an oscillation through an external mechanical device and then the moment of inertia around the rotational axis of the device is estimated using the equations of motion of the whole system using the input to the model and its response to the input.

The method proposed in this paper is based on the free oscillation technique. Its differences from methods previously proposed are as follows: 1) The model aircraft is suspended by two vertical lines directly without any suspension device; hence, there is neither aerodynamic nor mechanical interference from the device. Therefore, the moment of inertia can be measured without needing to eliminate the effects of general mass, added mass, and the dynamics of the device. 2) Furthermore, there is no need to reinforce and/or adapt the model itself to fit the external device geometrically and structurally. 3) In addition, we proposed a regression model that can be used to estimate all components of the virtual inertia matrix based on the body axes, using the multiple free oscillation test results.

\section{Experimental Method}

The model aircraft must be suspended by lines of the same length hanging from the ceiling or a certain horizontal rigid structure. The lighter, thinner, and stiffer the lines are, the smaller the influence of the lines on the measurement results is. Figure 1 shows the case where a small fixed-wing UAV is suspended by the two suspension lines attached at its wing tips. It is possible to apply this testing method to any size and type of object. It is nowadays common for unmanned aerial and underwater vehicles to be equipped with six-axis inertial measurement units (IMUs) consisting of three-axis gyroscopes and three-axis accelerometers; therefore, the IMUs are not considered additional devices in this experimental method, that is, there is no extra device nor equipment other than the suspension lines.

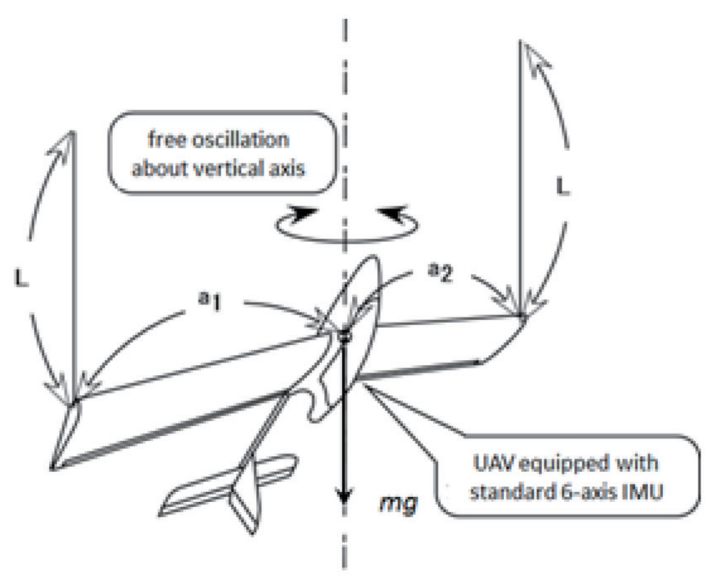

Fig. 1. Schematic of the free oscillation test for a fixed-wing UAV. 
The model is subject to a free rotational oscillation triggered by hand around the vertical axis, so that the moment of inertia around the vertical axis through the center of gravity $I_{v}$ can be estimated using

$$
I_{v}=\frac{a_{1} a_{2} m g}{\omega_{n}^{2} L},
$$

where $a_{1}$ and $a_{2}$ are the distances from the center of gravity to each of the two hook points, $m$ is the general mass of the model, $g$ is the acceleration due to gravity, $L$ is the length of the two suspending lines, and $\omega_{n}$ is the undamped natural circular frequency of the free rotational oscillation calculated from

$$
\omega_{n}=\sqrt{(2 \pi f)^{2}+n^{2}} .
$$

Here, $f$ is the frequency of the oscillation and $n$ is the damping coefficient. The frequency $f$ is analyzed using the waveforms of the signals from the gyroscopes. The damping coefficient $n$ is calculated as

$$
n=-f \cdot \delta,
$$

where $\delta$ is the logarithmic decrement of the signal waveforms and can be calculated as a natural logarithm of the ratio of successive peaks. Then, Eq. (2) can be represented as

$$
\omega_{n}=2 \pi f \cdot \sqrt{1+\left(\frac{\delta}{2 \pi}\right)^{2}} .
$$

In the case of fixed-wing vehicles, any rotational motion containing a rolling component (rotation around the fuselage axis, hereinafter defined as the $x$-axis of the body axes with its origin at the center of gravity) is typically heavily damped by the aerodynamic effect of the main wing; therefore, the logarithmic decrement cannot be neglected in Eq. (4). All three signals of angular velocities around the $x$-axis, $y$-axis (pointing toward the right wing), and $z$-axis (pointing downward) can be used to obtain the frequency $f$ and logarithmic decrement $\delta$ for calculating the undamped natural circular frequency $\omega_{n}$. It is advisable to choose the largest amplitude signal waveform or to take the average of the three results. For the purpose of estimating the natural frequency, there is no need to calibrate the gyroscopes and the raw data can be used in the post processing. A translational motion, which may happen unexpectedly, is regarded to be independent of rotational motions, and hence, will not affect the accuracy of the natural circular frequency.

Equation (1) gives the moment of inertia around the vertical axis at a certain attitude angle; however, the final goal is to obtain all components of the moments and products of inertia in the body axes with the added mass effect. The entire procedure of the free oscillation tests at 
different attitude angles is repeated by changing the combination of the two hook points so that the moments of inertia around different axes can be estimated using the multivariate regression model introduced in the following paragraph.

The moments and products of inertia in the body axes are defined as

$$
\begin{gathered}
I_{x x}=\int\left(y^{2}+z^{2}\right) d m, I_{y y}=\int\left(z^{2}+x^{2}\right) d m, I_{z z}=\int\left(x^{2}+y^{2}\right) d m, \\
I_{x y}=I_{y x}=\int x y d m, I_{x z}=I_{z x}=\int x z d m, I_{y z}=I_{z y}=\int y z d m,
\end{gathered}
$$

where $x, y$, and $z$ are the body-axis coordinates of the differential mass element $d m$ of the model and all of the above parameters can be represented in the form of the inertia matrix as

$$
\boldsymbol{I}_{B}=\left[\begin{array}{ccc}
I_{x x} & -I_{x y} & -I_{z x} \\
-I_{x y} & I_{y y} & -I_{y z} \\
-I_{z x} & -I_{y z} & I_{z z}
\end{array}\right] .
$$

Here, the subscript $B$ is used to denote the body axes, while the moment of inertia around the vertical axis $I_{v}$ estimated using Eq. (1) is one of the components of the inertia matrix in the earth-fixed frame represented by

$$
\boldsymbol{I}_{E}=\left[\begin{array}{ccc}
\cdots & \cdots & \cdots \\
\cdots & \cdots & \cdots \\
\cdots & \cdots & I_{V}
\end{array}\right],
$$

where the subscript $E$ is used to denote the earth-fixed frame and the last element $I_{V}$ is the only parameter required for the estimation of $\boldsymbol{I}_{B}$ through regression analysis. It is measured in each free oscillation test to yield a set of data points from repeated measurements. The following equation holds between $\boldsymbol{I}_{B}$ and $\boldsymbol{I}_{E}{ }^{(12)}$

$$
\boldsymbol{I}_{E}=\boldsymbol{L}_{E B} \cdot \boldsymbol{I}_{B} \cdot \boldsymbol{L}_{B E}
$$

Here, $\boldsymbol{L}_{E B}$ and $\boldsymbol{L}_{B E}$ are the transformation matrices consisting of direction cosines of attitude angles (yaw angle $\psi$, pitch angle $\theta$, and roll angle $\phi$ ), which are represented as

$$
\begin{aligned}
\boldsymbol{L}_{E B} & =\left[\begin{array}{ccc}
\cos \theta \cos \psi & \sin \phi \sin \theta \cos \psi-\cos \phi \sin \psi & \cos \phi \sin \theta \cos \psi+\sin \phi \sin \psi \\
\cos \theta \sin \psi & \sin \phi \sin \theta \sin \psi+\cos \phi \cos \psi & \cos \phi \sin \theta \sin \psi-\sin \phi \cos \psi \\
-\sin \theta & \sin \phi \cos \theta & \cos \phi \cos \theta
\end{array}\right], \text { (10) } \\
\boldsymbol{L}_{B E} & =\left[\begin{array}{ccc}
\cos \theta \cos \psi & \cos \theta \sin \psi & -\sin \theta \\
\sin \phi \sin \theta \cos \psi-\cos \phi \sin \psi & \sin \phi \sin \theta \sin \psi+\cos \phi \cos \psi & \sin \phi \cos \theta \\
\cos \phi \sin \theta \cos \psi+\sin \phi \sin \psi & \cos \phi \sin \theta \sin \psi-\sin \phi \cos \psi & \cos \phi \cos \theta
\end{array}\right] . \text { (11) }
\end{aligned}
$$


The substitution of Eqs. (7), (8), (10), and (11) into Eq. (9) yields

$$
\begin{aligned}
I_{V}= & I_{x x}(\sin \theta)^{2}+I_{y y}(\sin \phi \cos \theta)^{2}+I_{z z}(\cos \phi \cos \theta)^{2}-I_{x y}(-2 \sin \theta \sin \phi \cos \theta) \\
& -I_{z x}(-2 \sin \theta \cos \phi \cos \theta)-I_{y z}(2 \sin \phi \cos \theta \cos \phi \cos \theta) .
\end{aligned}
$$

The yaw angle $\psi$ has vanished in Eq. (12). Under the stationary condition before and after the free oscillation test run, the accelerometers are regarded as the inclinometer so that the accelerometer outputs $\left(a_{x}, a_{y}, a_{z}\right)$ can be expressed as functions of the pitch angle $\theta$ and roll angle $\phi$ as

$$
\left(\begin{array}{l}
a_{x} \\
a_{y} \\
a_{z}
\end{array}\right)=\left(\begin{array}{c}
\sin \theta \\
-\sin \phi \cos \theta \\
-\cos \phi \cos \theta
\end{array}\right) .
$$

Substituting Eq. (13) back into Eq. (12), we obtain

$$
I_{V}=I_{x x} \cdot a_{x}^{2}+I_{y y} \cdot a_{y}^{2}+I_{z z} \cdot a_{z}^{2}-I_{z x} \cdot 2 a_{z} a_{x}-I_{x y} \cdot 2 a_{x} a_{y}-I_{y z} \cdot 2 a_{y} a_{z} .
$$

Now we regard the above equation as the one in which the moment of inertia around the vertical axis $I_{v}$ is calculated from three-axis accelerometer outputs and the moments and products of inertia in the body axes. Let Eq. (14) be simplified as

$$
y=I_{x x} \cdot x_{1}+I_{v v} \cdot x_{2}+I_{z z} \cdot x_{3}+I_{z x} \cdot x_{4}+I_{x v} \cdot x_{5}+I_{v z} \cdot x_{6} \text {, }
$$

where

$$
\begin{gathered}
y=I_{V}, \\
\left(\begin{array}{llllllll}
x_{1} & x_{2} & x_{3} & x_{4} & x_{5} & x_{6}
\end{array}\right)=\left(\begin{array}{llllll}
a_{x}^{2} & a_{y}{ }^{2} & a_{z}^{2} & -2 a_{z} a_{x} & -2 a_{x} a_{y} & -2 a_{y} a_{z}
\end{array}\right),
\end{gathered}
$$

are defined as the objective variable $y$ and the explanatory variables $x_{1}$ to $x_{6}$, respectively, in the interest of simplicity in order to derive the following regression equation. We assume here that the free oscillation tests are repeated $N$ times at different attitude angles by changing either or both of the two hook points on the model and then the objective variables $y_{j}$ and the explanatory variables $x_{i j}(i=1$ to $6, j=1$ to $N$ ) are obtained. Now we rewrite Eq. (15) as

$$
\boldsymbol{y}=\boldsymbol{X} \cdot \boldsymbol{b},
$$

where the vectors $\boldsymbol{y}$ and $\boldsymbol{b}$ and the matrix $\boldsymbol{X}$ are

$$
\boldsymbol{y}=\left(\begin{array}{llll}
y_{1} & y_{2} & \ldots & y_{N}
\end{array}\right)^{\mathrm{T}}
$$




$$
\begin{aligned}
& \boldsymbol{b}=\left(\begin{array}{llllll}
I_{x x} & I_{y y} & I_{z z} & I_{z x} & I_{x y} & I_{y z}
\end{array}\right), \\
& \boldsymbol{X}=\left(\begin{array}{cccc}
x_{11} & x_{21} & \cdots & x_{61} \\
x_{12} & x_{22} & \cdots & x_{62} \\
\vdots & \vdots & \ddots & \vdots \\
x_{1 N} & x_{2 N} & \cdots & x_{6 N}
\end{array}\right)
\end{aligned}
$$

Here, the number of oscillation tests, $N$, should be greater than 6 or greater than 4 when $I_{x y}$ and $I_{y z}$ are assumed to be zero. The vector $\mathrm{b}$ consisting of the moments and products of inertia can be estimated as

$$
\boldsymbol{b}=\left(\boldsymbol{X}^{\mathrm{T}} \cdot \boldsymbol{X}\right)^{-1} \cdot\left(\boldsymbol{X}^{\mathrm{T}} \cdot \boldsymbol{y}\right)
$$

This matrix equation can be expanded to give

$$
\left(\begin{array}{l}
I_{x x} \\
I_{y y} \\
I_{z z} \\
I_{z x} \\
I_{x y} \\
I_{y z}
\end{array}\right)=\left\{\sum_{j=1}^{N}\left[\begin{array}{cccccc}
x_{1 j} x_{1 j} & x_{1 j} x_{2 j} & x_{1 j} x_{3 j} & x_{1 j} x_{4 j} & x_{1 j} x_{5 j} & x_{1 j} x_{6 j} \\
x_{2 j} x_{1 j} & x_{2 j} x_{2 j} & x_{2 j} x_{3 j} & x_{2 j} x_{4 j} & x_{2 j} x_{5 j} & x_{2 j} x_{6 j} \\
x_{3 j} x_{1 j} & x_{3 j} x_{2 j} & x_{3 j} x_{3 j} & x_{3 j} x_{4 j} & x_{3 j} x_{5 j} & x_{3 j} x_{6 j} \\
x_{4 j} x_{1 j} & x_{4 j} x_{2 j} & x_{4 j} x_{2 j} & x_{4 j} x_{2 j} & x_{4 j} x_{2 j} & x_{4 j} x_{2 j} \\
x_{5 j} x_{1 j} & x_{5 j} x_{2 j} & x_{5 j} x_{3 j} & x_{5 j} x_{4 j} & x_{5 j} x_{5 j} & x_{5 j} x_{6 j} \\
x_{6 j} x_{1 j} & x_{6 j} x_{2 j} & x_{6 j} x_{3 j} & x_{6 j} x_{4 j} & x_{6 j} x_{5 j} & x_{6 j} x_{6 j}
\end{array}\right]\right\}^{-1}\left\{\sum_{j=1}^{N}\left(\begin{array}{c}
x_{1 j} y_{j} \\
x_{2 j} y_{j} \\
x_{3 j} y_{j} \\
x_{4 j} y_{j} \\
x_{5 j} y_{j} \\
x_{6 j} y_{j}
\end{array}\right)\right\} .
$$

Since most aerial vehicles are very nearly symmetrical, it is common to assume them to be totally symmetrical in the design and analysis of the flight control system. It then becomes possible to reduce the dimension of the matrix in Eq. (24), as shown in

$$
\left(\begin{array}{l}
I_{x x} \\
I_{y y} \\
I_{z z} \\
I_{z x}
\end{array}\right)=\left\{\sum_{j=1}^{N}\left[\begin{array}{cccc}
x_{1 j} x_{1 j} & x_{1 j} x_{2 j} & x_{1 j} x_{3 j} & x_{1 j} x_{4 j} \\
x_{2 j} x_{1 j} & x_{2 j} x_{2 j} & x_{2 j} x_{3 j} & x_{2 j} x_{4 j} \\
x_{3 j} x_{1 j} & x_{3 j} x_{2 j} & x_{3 j} x_{3 j} & x_{3 j} x_{4 j} \\
x_{4 j} x_{1 j} & x_{4 j} x_{2 j} & x_{4 j} x_{2 j} & x_{4 j} x_{4 j}
\end{array}\right]\right\}^{-1}\left\{\sum_{j=1}^{N}\left(\begin{array}{l}
x_{1 j} y_{j} \\
x_{2 j} y_{j} \\
x_{3 j} y_{j} \\
x_{4 j} y_{j}
\end{array}\right)\right\} .
$$

Equation (25) gives the best fit for a set of $I_{x x}, I_{y y}, I_{z z}$, and $I_{z x}$ using the sample data sets $y_{i}$ and $x_{i j}(i=1$ to $6, j=1$ to $N$ ) based on the measured attitude angles (pitching angle $\theta$ and rolling angle $\phi$ ) under stationary conditions and the frequency $f$ and logarithmic decrement $\delta$ under oscillatory conditions. In general, the greater the number of oscillation tests, the more accurate the regression analysis. However, it is even more important to plan the sequential runs of oscillation tests in an efficient manner, that is, to change $x_{1}, x_{2}, x_{3}, x_{4}, x_{5}$, and $x_{6}$ as independently as possible. 
We propose to carry out two sets of oscillation test runs, rolling and pitching test runs, as an efficient way of changing the explanatory variables $x_{1}, x_{2}, x_{3}, x_{4}, x_{5}$, and $x_{6}$. The positional relationships of the hook points against the center of gravity of the model determine the attitude angles, which are the roll angle $\phi$ and pitch angle $\theta$. In the rolling test run, the fore and aft locations of the lower part of the fuselage of the model are used as the hook points, where it is likely that the model is suspended under the reversed condition (upside down) and the rolling angles can be varied by repositioning either of or both the two hook points from side to side as illustrated in Fig. 2. The supporting points on the ceiling, the hook points, and the center of gravity of the model should align into a straight line. Here, $x_{2}, x_{3}, x_{5}$, and $x_{6}$ are mainly varied so that $I_{y y}, I_{z z}, I_{x y}$, and $I_{y z}$ can be estimated. On the other hand, in the pitching test run, the wing tips are used as the hook points, where the pitch angles can be varied by repositioning the longitudinal (chordwise) locations of the two hook points at the wing tips. Here, $x_{1}, x_{3}$, and $x_{4}$ are mainly varied so that $I_{x x}, I_{z z}$, and $I_{z x}$ can be estimated, as illustrated in Fig. 3. The rolling and pitching test runs can be combined by properly choosing an asymmetric layout of the two hook points, yielding more robust regression analysis results because of the less correlated distribution of explanatory variables. Slight changes in the positions of the hook points are effective for inducing sufficient changes in both roll and pitch angles.
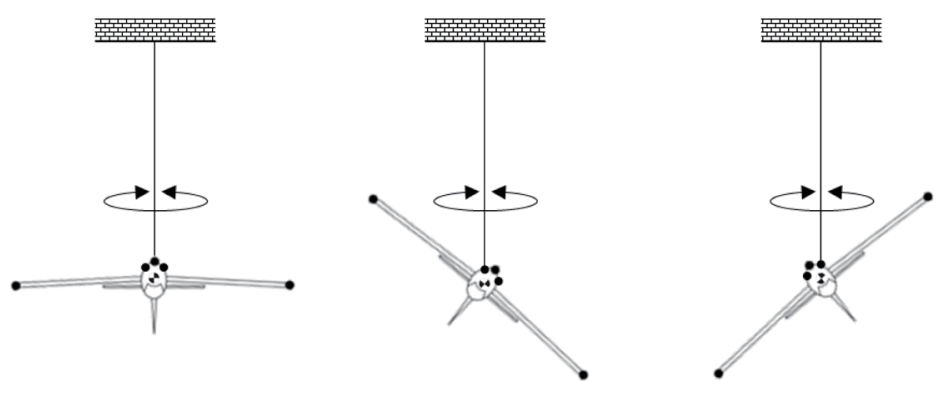

Fig. 2. Schematics of front views of the UAV model for the rolling test run. Rolling angles: 0 deg (left), $+135 \mathrm{deg}$ (center), and $-135 \mathrm{deg}$ (right).
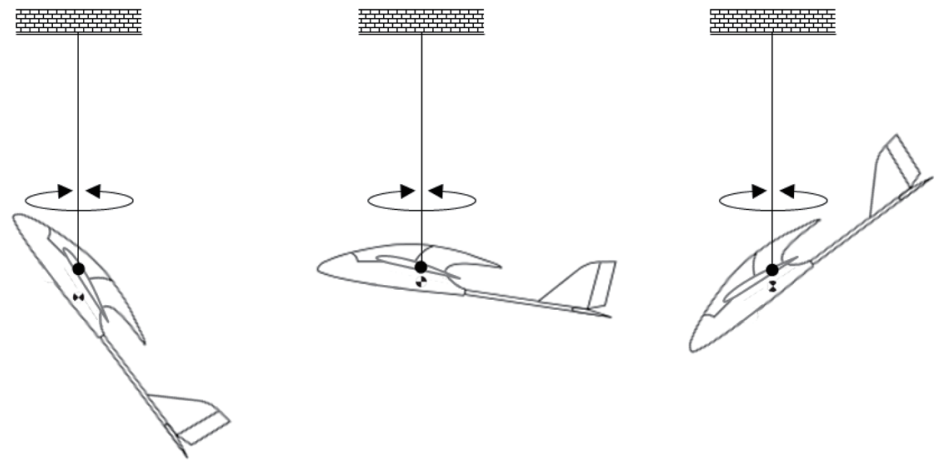

Fig. 3. Schematics of left-side views of the UAV model for the pitching test run. Pitch angles: 0 deg (left), $+135 \mathrm{deg}$ (center), and $-135 \mathrm{deg}$ (right). 


\section{Experimental Results}

To investigate the effectiveness of the experimental method proposed in the previous section, a small fixed-wing UAV with a total mass of $1.391 \mathrm{~kg}$, a wing span of $1.360 \mathrm{~m}$, and a chord length of $0.228 \mathrm{~m}$ was tested. The top view of the UAV model is shown in Fig. 4. The hook points are located at the forward and backward locations at the bottom of the fuselage (F1, F2, F3, B1, B2, and B3) and both tip ends (L1, L2, L3, L4, R1, R2, R3, and R4). The theoretical estimation of the added inertia moment about the $x$-axis is approximately $30 \%$ of the general inertia moment; therefore, it is important to measure the virtual inertia matrix experimentally.

The experimental procedure is as follows:

[1] Measure the length of the suspension lines, $L$, the mass $m$, and the center of gravity of the model to calculate the distances between the center of gravity and the hook points $a_{1}$ and $a_{2}$.

[2] Choose two hook points and hang the model from the ceiling or a rigid structure using the suspension lines through the hook points.

[3] Measure the three-axis accelerometer outputs $a_{x}, a_{y}$, and $a_{z}$ under the stationary condition and convert them into $x_{i j}$ using Eq. (17).

[4] Apply rotational oscillation by hand around the vertical axis and measure the three-axis gyroscope outputs $\omega_{x}, \omega_{y}$, and $\omega_{z}$ under the oscillatory condition.

[5] Analyze the signal of the gyroscope outputs to obtain the dominant frequency $f$ and logarithmic decrement $\delta$.

[6] Calculate the undamped natural circular frequency $\omega_{n}$ using Eq. (4).

[7] Calculate the virtual moment of inertia around the vertical axis through the center of gravity, $y_{j}=I_{\nu}$, using Eqs. (1) and (16).

[8] Repeat the above steps from [1] to [6] $N$ times at different attitude angles $(j=1$ to $N)$.

[9] Analyze all components of the virtual inertia matrix, $I_{x x}, I_{y y}, I_{z z}, I_{z x}, I_{x y}$, and $I_{y z}$, using Eqs. (24) and/or (25). Equation (25) ignores $I_{x y}$ and $I_{y z}$ to obtain the best fit for $I_{x x}, I_{y y}, I_{z z}$, and $I_{z x}$ in the case of the model having the $x-z$ plane of symmetry, while Eq. (24) can still be
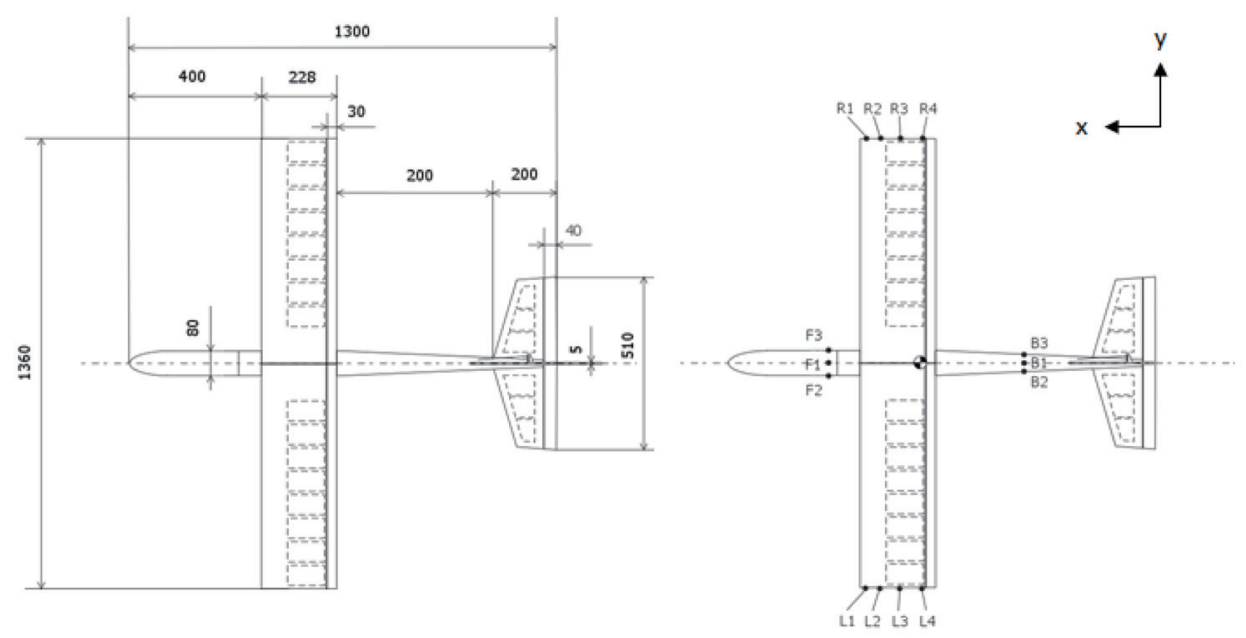

Fig. 4. Main dimensions and hook points of the UAV model tested. 
effectively used to see how precisely the measurement is carried out by checking the two results from Eqs. (24) and (25).

Table 1 shows the results of the measurements using accelerometers and gyroscopes in both rolling and pitching test runs. It is observed that the roll angles are mainly varied for the rolling test runs, while the pitch angles are mainly varied for the pitching test runs, and that the frequencies are more varied for the pitching test runs than for the rolling test runs. Table 2

Table 1

Results of measurements using three-axis accelerometers and gyroscopes in both rolling and pitching test runs.

\begin{tabular}{|c|c|c|c|c|c|c|c|c|c|c|}
\hline Exp. No. & & & $\begin{array}{c}A_{B X} \\
(\mathrm{G})\end{array}$ & $\begin{array}{l}A_{B Y} \\
(\mathrm{G})\end{array}$ & $\begin{array}{c}A_{B Z} \\
(\mathrm{G})\end{array}$ & $\begin{array}{c}\phi_{B} \\
(\mathrm{deg})\end{array}$ & $\begin{array}{c}\theta_{B} \\
(\mathrm{deg})\end{array}$ & $\begin{array}{c}f \\
(\mathrm{~Hz})\end{array}$ & $\begin{array}{c}n \\
(\mathrm{rad} / \mathrm{sec})\end{array}$ & $\begin{array}{c}\omega_{n} \\
(\mathrm{rad} / \mathrm{sec})\end{array}$ \\
\hline Roll-1 & F1 & B1 & -0.006 & 0.055 & 0.977 & -176.8 & -0.4 & 0.307 & -0.023 & 1.930 \\
\hline Roll-2 & $\mathrm{F} 2$ & B2 & -0.030 & 0.898 & 0.477 & -118.0 & -1.7 & 0.349 & -0.037 & 2.193 \\
\hline Roll-3 & F3 & B3 & -0.074 & -0.824 & 0.532 & 122.9 & -4.2 & 0.351 & -0.032 & 2.207 \\
\hline Roll-4 & F2 & B1 & 0.019 & 0.525 & 0.869 & -148.8 & 1.1 & 0.322 & -0.016 & 2.020 \\
\hline Roll-5 & F1 & B2 & -0.057 & 0.387 & 0.916 & -157.1 & -3.3 & 0.315 & -0.019 & 1.980 \\
\hline Roll-6 & F3 & B1 & 0.010 & -0.407 & 0.900 & 155.7 & 0.6 & 0.321 & -0.020 & 2.016 \\
\hline Roll-7 & F1 & B3 & -0.118 & -0.280 & 0.949 & 163.6 & -6.8 & 0.314 & -0.019 & 1.976 \\
\hline Pitch-1 & $\mathrm{R} 1$ & L1 & 0.927 & -0.028 & -0.424 & 3.8 & 67.9 & 0.999 & -0.060 & 6.279 \\
\hline Pitch-2 & R2 & L2 & 0.864 & -0.028 & -0.531 & 3.1 & 59.8 & 0.933 & -0.066 & 5.861 \\
\hline Pitch-3 & R3 & L3 & 0.691 & -0.029 & -0.721 & 2.3 & 43.7 & 0.827 & -0.041 & 5.196 \\
\hline Pitch-4 & $\mathrm{R} 4$ & L4 & -0.114 & -0.029 & -0.999 & 1.7 & -6.6 & 0.707 & -0.022 & 4.442 \\
\hline Pitch-5 & $\mathrm{R} 1$ & L2 & 0.715 & -0.146 & -0.694 & 11.9 & 45.7 & 0.857 & -0.042 & 5.384 \\
\hline Pitch-6 & $\mathrm{R} 1$ & L3 & 0.640 & -0.093 & -0.768 & 6.9 & 39.8 & 0.803 & -0.039 & 5.043 \\
\hline Pitch-7 & $\mathrm{R} 1$ & L4 & 0.408 & -0.057 & -0.930 & 3.5 & 24.1 & 0.738 & -0.030 & 4.640 \\
\hline Pitch-8 & $\mathrm{R} 2$ & L1 & 0.751 & 0.066 & -0.672 & -5.6 & 48.7 & 0.863 & -0.041 & 5.420 \\
\hline Pitch-9 & R3 & L2 & 0.627 & 0.022 & -0.786 & -1.6 & 38.8 & 0.801 & -0.043 & 5.034 \\
\hline Pitch-10 & $\mathrm{R} 4$ & L3 & 0.399 & -0.020 & -0.922 & 1.2 & 23.5 & 0.741 & -0.031 & 4.657 \\
\hline
\end{tabular}

Table 2

Explanatory variables $x_{i j}$ and objective variables $y_{j}$ calculated from the measurement results.

\begin{tabular}{|c|c|c|c|c|c|c|c|c|c|}
\hline Exp. No. & & & $\begin{array}{c}x_{1} \\
\left(\mathrm{G}^{2}\right)\end{array}$ & $\begin{array}{c}x_{2} \\
\left(\mathrm{G}^{2}\right)\end{array}$ & $\begin{array}{c}x_{3} \\
\left(G^{2}\right)\end{array}$ & $\begin{array}{c}x_{4} \\
\left(G^{2}\right)\end{array}$ & $\begin{array}{c}x_{5} \\
\left(G^{2}\right)\end{array}$ & $\begin{array}{c}x_{6} \\
\left(\mathrm{G}^{2}\right)\end{array}$ & $\begin{array}{c}y \\
\left(\mathrm{kgm}^{2}\right)\end{array}$ \\
\hline Roll-1 & F1 & B1 & 0.000 & 0.003 & 0.954 & 0.012 & 0.001 & -0.108 & 0.167 \\
\hline Roll-2 & $\mathrm{F} 2$ & B2 & 0.001 & 0.806 & 0.227 & 0.029 & 0.054 & -0.856 & 0.130 \\
\hline Roll-3 & F3 & B3 & 0.005 & 0.679 & 0.283 & 0.079 & -0.122 & 0.877 & 0.128 \\
\hline Roll-4 & $\mathrm{F} 2$ & B1 & 0.000 & 0.276 & 0.755 & -0.032 & -0.020 & -0.912 & 0.153 \\
\hline Roll-5 & $\mathrm{F} 1$ & B2 & 0.003 & 0.150 & 0.839 & 0.105 & 0.044 & -0.708 & 0.159 \\
\hline Roll-6 & F3 & B1 & 0.000 & 0.166 & 0.811 & -0.019 & 0.009 & 0.734 & 0.154 \\
\hline Roll-7 & $\mathrm{F} 1$ & B3 & 0.014 & 0.078 & 0.901 & 0.224 & -0.066 & 0.532 & 0.160 \\
\hline Pitch-1 & $\mathrm{R} 1$ & L1 & 0.859 & 0.001 & 0.180 & 0.785 & 0.052 & -0.024 & 0.084 \\
\hline Pitch-2 & $\mathrm{R} 2$ & L2 & 0.747 & 0.001 & 0.282 & 0.918 & 0.049 & -0.030 & 0.096 \\
\hline Pitch-3 & R3 & L3 & 0.478 & 0.001 & 0.519 & 0.996 & 0.040 & -0.042 & 0.122 \\
\hline Pitch-4 & $\mathrm{R} 4$ & L4 & 0.013 & 0.001 & 0.998 & -0.228 & -0.007 & -0.058 & 0.168 \\
\hline Pitch-5 & $\mathrm{R} 1$ & L2 & 0.512 & 0.021 & 0.482 & 0.993 & 0.209 & -0.203 & 0.114 \\
\hline Pitch-6 & $\mathrm{R} 1$ & L3 & 0.410 & 0.009 & 0.590 & 0.983 & 0.119 & -0.142 & 0.130 \\
\hline Pitch-7 & $\mathrm{R} 1$ & L4 & 0.166 & 0.003 & 0.865 & 0.759 & 0.047 & -0.106 & 0.154 \\
\hline Pitch-8 & $\mathrm{R} 2$ & L1 & 0.564 & 0.004 & 0.451 & 1.009 & -0.099 & 0.088 & 0.113 \\
\hline Pitch-9 & R3 & L2 & 0.393 & 0.000 & 0.618 & 0.985 & -0.028 & 0.035 & 0.130 \\
\hline Pitch-10 & $\mathrm{R} 4$ & L3 & 0.159 & 0.000 & 0.850 & 0.736 & 0.016 & -0.037 & 0.152 \\
\hline
\end{tabular}




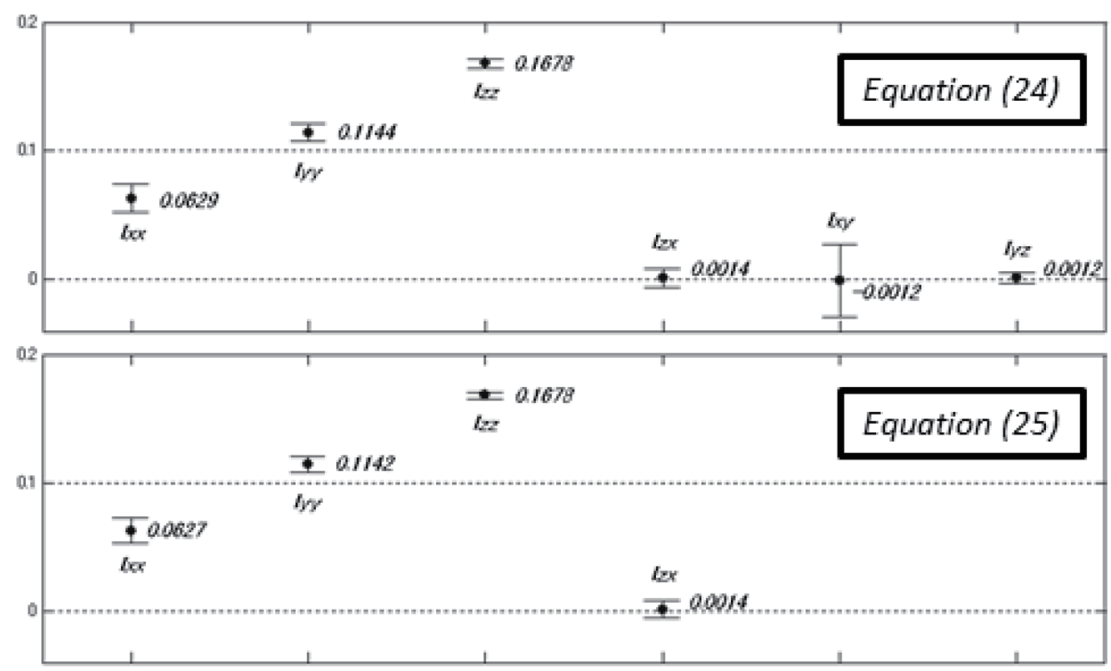

Fig. 5. Estimates and $95 \%$ confidence intervals of the virtual inertia moments and products: the results obtained considering all components of the virtual inertia matrix (top) and those obtained ignoring $I_{x y}$ and $I_{y z}$ (bottom).

shows the variations of the explanatory variables $x_{i j}$ and objective variables $y_{i}$ calculated from the measurement results, to be used for the multivariate regression analysis using Eqs. (24) and (25).

Figure 5 shows the estimates and $95 \%$ confidence intervals of the virtual inertia moments and products both obtained considering all components of the virtual inertia matrix and ignoring $I_{x y}$ and $I_{y z}$. There are good agreements in $I_{x x}, I_{y y}, I_{z z}$, and $I_{z x}$ between the results from Eqs. (24) and (25), and furthermore, the results of $I_{x y}$ and $I_{y z}$ estimated from Eq. (24) are almost zero, which means that the measurements and regression analysis proposed in this paper can provide highly accurate results.

\section{Conclusions}

In this paper, a new method of estimating the virtual inertia matrix, which can be most effectively applied to small UAVs, was presented. Here, the model was directly suspended vertically using two thin light weight low-stretch lines and subject to free rotational oscillations around the vertical axis at different attitude angles so that all components of the virtual inertia matrix can be estimated using multivariate regression models. This method, including postprocessing using regression analysis, is characterized by 1) experimental simplicity - there is no need to adapt the model to any external devices, and therefore, there is no mechanical interference from experimental devices to the measurement results, and 2) accuracy - the measurement results include all components of the inertia matrix based on the body axes with the added mass effect without aerodynamic interference from any external devices. The method proposed in this paper can provide all the inertia characteristics required for the design and simulation of flight control systems. 


\section{References}

1 C. Schedlinski and M. Link: Mech. Syst. Sig. Process. 15 (2001) 189. https://doi.org/10.1006/mssp.2000.1345

2 S. A. Hartley and M. P. Marbel: NACA Report, No. 467 (1934) 501. https://ntrs.nasa.gov/archive/nasa/casi.ntrs. nasa.gov/19930091541.pdf

3 M. Yanagihara, M. Nagayasu, S. Sasa, and T. Shimomura: NAL-TM-641 (1991). https://repository.exst.jaxa.jp/ dspace/bitstream/a-is/25901/1/naltm00641.pdf

4 M. R. Jardin and E. R. Mueller: J. Aircraft 46 (2007) 763. https://doi.org/10.2514/1.34015

5 A. Halder, V. Agarwal, R.Garhwal, and M Sinha: https://www.abhishekhalder.org/published_IEI_ Inertial_2009.pdf (accessed September 2019).

6 G. Previati, M. Gobbi, and G. Mastinu: J. Aircraft 49 (2012) 444. https://doi.org/10.2514/1.C031369

7 K. Lehmkuhler, K. C. Wong, and D. Verstraete: Aeronaut. J. 120 (2016) 1785. https://doi.org/10.1017/ aer.2016.105

8 O. D. Dantsker, M. Vahora, S. Imtiaz, and M. Caccamo: AIAA 2018-4219 (2018). https://doi. org/10.2514/6.2018-4219

9 R. C. de Jong and J. A. Mulder: J. Aircraft 24 (1987) 362. https://doi.org/10.2514/3.45454

10 H. Ohta and H. Nishimura: J. Jpn. Soc. Aeronaut. Space Sci. 42 (1994) 108. https://doi.org/10.2322/ jjsass1969.42.108

11 S. Higashino, M. Matsubara, and A. Sakurai: J. Jpn. Soc. Aeronaut. Space Sci. 45 (1997) 79. https://doi. org/10.2322/jjsass1969.45.79

12 B. Etkin and L. D. Reid: Dynamics of Flight, Stability and Control (John Wiley \& Sons, Inc., New York, 1995) 3rd ed., p. 315. 